\title{
EXISTENCE OF POSITIVE AND Ф-BOUNDED HARMONIC FUNCTIONS ON RIEMANNIAN MANIFOLDS
}

\author{
WELLINGTON H. OW
}

1. By a Riemannian manifold $R$ we mean a connected, orientable $C^{\infty}$-manifold of dimension $n \geqq 2$ possessing a $C^{\infty}$-metric tensor. Let $\Phi(t)$ be any nonnegative real-valued function defined on $[0, \infty)$. A harmonic function $u$ on $R$ is said to be $\Phi$-bounded on $R$ if the composite function $\Phi(|u|)$ possesses a harmonic majorant on $R$. We denote by $H \Phi(R)$, or simply $H \Phi$, the class of all $\Phi$-bounded harmonic functions on $R$ and by $O_{H \Phi}$ the null class consisting of all Riemannian manifolds $R$ on which every $\Phi$-bounded harmonic function reduces to a constant. The problem of classifying Riemann surfaces with respect to $O_{H \Phi}$ was first attempted by Parreau [4] for the special case where $\Phi$ was increasing and convex. Later Nakai [1] completely determined $O_{H \Phi}$ for general $\Phi$ in the 2-dimensional case. Recently $\mathrm{Ow}$ [3] extended the $\Phi$-bounded notion to harmonic spaces and determined $O_{H \Phi}$ there. In his paper mentioned above Nakai also considered the classification of Riemann surfaces with regular boundaries. In this investigation he partially characterized the class $S O_{H \Phi}$, where a subsurface $G$ with regular boundary is said to belong to $S O_{H \Phi}$ if every $\Phi$-bounded harmonic function on $G$ which vanishes continuously on $\partial G$ is identically zero.

The purpose of this paper is to determine the class $\mathrm{SO}_{H \Phi}$ completely for the 2-dimensional (i.e. Riemann surface) as well as for the higher dimensional cases. An important factor in this regard is a theorem of Parreau on the existence of positive harmonic functions. In higher dimensions the class of admissible subregions will consist of smooth subregions of Riemannian $n$-manifolds. Here a subregion $G$ of $R$ will be called smooth if its relative boundary $\partial G \neq \varnothing$ satisfies the following: Each point $p \in \partial G$ has a neighborhood $N$ and a diffeomorphism $h$ of $N$ with a region in $E^{n}$ such that $h(N \cap \partial G)$ is contained in a hyperplane.

2. Before giving a characterization of $\mathrm{SO}_{H \Phi}$ we shall first give some necessary preliminary results. The following theorem of Parreau [4],

Received by the editors June 28, 1971.

AMS (MOS) subject classifications (1970). Primary 31A05, 31B05, 30A48; Secondary 31A15, 31 B15. 
proven for the 2-dimensional case, is also valid in the higher dimensional case:

THEOREM 1 . If $G$ is any smooth subregion with noncompact closure $\bar{G}$ then there exists a nonconstant positive harmonic function $u$ on $G$ with continuous boundary value zero on $\partial G$.

A simple proof of the above theorem for Riemann surfaces has also been given by Nakai [2]. The proof of the theorem for general Riemannian manifolds will be given in $\$ 4$.

3. Denote by $H P(G)$ the class of nonnegative harmonic functions on a subregion $G \subset R$. Let $G \subset R$ be smooth and $\left\{u_{n}\right\} \subset H P(G)$ a sequence such that $u=\lim _{n} u_{n}$ exists in $G$. Then we have the following counterpart of Nakai's result (cf. [5] ):

Lemma 1. If each $u_{n}$ is continuous on $G \cup \partial G$ and $u_{n} \mid \partial G=0$ then $u$ is continuous on $G \cup \partial G$ and vanishes continuously on $\partial G$.

Proof. Let $p_{0} \in \partial G$ and $B$ be an open parametric ball about $p_{0}$ chosen so that $B \cap G$ is itself a relatively compact smooth ball about $p_{0}$ with boundary $\partial(B \cap G)$ consisting of $(\partial B) \cap G$ and $(\partial G) \cap \bar{B}$. Denote by $g(p, a)$ the Green's function for $B \cap G$ with pole $a \in B \cap G$. Define a regular Borel measure $\mu_{n}$ on $\partial(B \cap G)$ by $d \mu_{n}(p)=$ $u_{n}(p) d S(p)$ where $d S(p)$ denotes the surface element on $\partial(B \cap G)$. Now

$$
u_{n}(a)=-\int_{\partial(B \cap G)} u_{n}(p) \frac{\partial g(p, a)}{\partial n} d \mathrm{~S}(p)
$$

where $\partial g / \partial n$ is the normal derivative defined in terms of the Hodge star operator * by $(\partial g / \partial n) d \mathrm{~S}=* d g$. It is well known that $-\partial g(p, a) / \partial n$ is continuous and positive, and therefore

$$
\inf _{\partial(B \cap G)}-\frac{\partial g(p, a)}{\partial n}=m>0 .
$$

Hence

$$
u_{n}(a) \geqq m \int_{\partial(B \cap G)} d \mu_{n}(p)
$$

and consequently $\mu_{n}(\partial(B \cap G)) \leqq u_{n}(a) / m$. As a result the sequence $\left\{\mu_{n}(\partial(B \cap G))\right\}$ is bounded and, hence, by the selection theorem there exists a regular Borel measure $\mu(p)$ such that

$$
\lim _{n} \int_{\partial(B \cap G)} \lambda(p) d \mu_{n}(p)=\int_{\partial(B \cap G)} \lambda(p) d \mu(p)
$$


for any real-valued continuous function $\lambda$ on $\partial(B \cap G)$. In particular if $\lambda(p)=-\partial g(p, a) / \partial n$ we have from (1) that

$$
u(a)=-\int_{\partial(B \cap G)} \frac{\partial g(p, a)}{\partial n} d \mu(p) .
$$

Now $u_{n} \mid(\partial G) \cap \bar{B}=0$, and so $\mu_{n}((\partial G) \cap \bar{B})=0$. Consequently $\mu((\partial G) \cap \bar{B})=0$ and hence

$$
u(a)=-\int_{(\partial B) \cap G} \frac{\partial g(p, a)}{\partial n} d \mu(p) .
$$

Finally since $\lim _{a \rightarrow \partial G} \partial g(p, a) / \partial n=0$ it follows that $\lim _{a \rightarrow \partial G} u(a)=0$ as asserted.

4. Proof of Theorem 1. Let $p_{0} \in G$ be fixed and $\left\{p_{n}\right\}$ a sequence of points in $G$ converging to the ideal boundary of $G$, i.e. the Alexandroff compactification point. Set

$$
u_{n}(p)=g\left(p, p_{n}\right) / g\left(p_{0}, p_{n}\right),
$$

where $p \in G-p_{n}$ and $g\left(p, p_{n}\right)$ is the Green's function for $G$. Since $u_{n}\left(p_{0}\right)=1$ and $u_{n}>0$ there exists a convergent subsequence, again denoted by $\left\{u_{n}\right\}$. Let $u=\lim _{n} u_{n}$. Then $u\left(p_{0}\right)=1$ and $u>0$ on $G$. By Lemma $1, u$ vanishes continuously on $\partial G$.

5. If $G \subset R$ is a smooth subregion we say that $G \in S O_{H P}$ provided that every $u \in H P(G)$ which vanishes continuously on $\partial G$ is identically zero on $G$. For any smooth subregion $G \subset R$ we denote by $H_{0} \Phi=H_{0} \Phi(R, G)$ the class of harmonic functions $u$ on $G$ vanishing continuously on $\partial G$ and such that $\Phi(|u|)$ possesses a harmonic majorant on $G$. Here $\Phi$ is an arbitrary nonnegative real-valued function defined on $[0, \infty)$. The corresponding null class $S O_{H \Phi}$ will consist of those smooth subregions $G$ for which $H_{0} \Phi=\{0\}$. We observe that if $\Phi$ is not bounded in any neighborhood of $t=0$ then $\mathrm{SO}_{H \Phi}$ consists of all smooth subregions $G \subset R$. On the other hand suppose $\Phi$ is bounded in some neighborhood of $t=0$. Denote by $S O_{H B}$ the class of smooth subregions $G \subset R$ such that every bounded harmonic function which vanishes continuously on $\partial G$ is identically zero on $G$. We then have

THEOREM 2. (a) If $\Phi$ is not bounded in some finite neighborhood of $t=0$ then $\mathrm{SO}_{H \Phi}=\mathrm{SO}_{H B}$.

(b) If $\Phi$ is bounded in any finite neighborhood of $t=0$ then $S O_{H \Phi}=S O_{H P}\left(S O_{H \Phi}=S O_{H B}\right)$ provided that lim $\sup _{t \rightarrow \infty} \Phi(t) / t<\infty$ $\left(\lim \sup _{t \rightarrow \infty} \Phi(t) / t=\infty\right)$. 
Before proving Theorem 2 we state

LEMMA 2. The class $\mathrm{SO}_{H P}$ consists of all relatively compact smooth subregions of $R$.

Proof. Clearly any relatively compact smooth subregion belongs to $S O_{H P}$. By Theorem 1, it follows that $S O_{H P}$ consists precisely of relatively compact smooth subregions.

6. Proof of Theorem 2. We first prove part (a). By assumption $\Phi(t) \leqq c=$ const for $t \leqq t_{0}$. If $u$ is a nonconstant bounded harmonic function on $G$ vanishing continuously on $\partial G$, then, upon multiplication by a suitable constant $c_{0}$, we have $\left|c_{0} u\right| \leqq t_{0}$ and hence $\Phi\left(\left|c_{0} u\right|\right) \leqq c$. Therefore, $\mathrm{SO}_{H \Phi} \subset S O_{H B}$. In order to obtain $\mathrm{SO}_{H B} \subset$ $\mathrm{SO}_{H \Phi}$ we note that by assumption there exists a neighborhood $\left[0, t^{*}\right]$ such that $\sup _{0 \leqq t \leqq t^{*}} \Phi(t)=\infty$. Clearly $u<t^{*}$. This completes the proof of part (a).

We next prove part (b). First assume that $\lim \sup _{t \rightarrow \infty} \Phi(t) / t<\infty$ and $\Phi$ is bounded in any finite neighborhood of $t=0$. It follows that there exist constants $c_{1}$ and $c_{2}$ such that $\Phi(t) \leqq c_{1}+c_{2} t$ on $[0, \infty)$. Therefore, every $H P$-function is an $H \Phi$-function. This remark together with Lemma 2 proves the first half of part (b). A proof of the remaining part can be found in [3]. This completes the proof of Theorem 2.

Summarizing these results diagramatically:

$\Phi$ unbounded in any
neighborhood of $t=0: S O_{H \Phi}=\{$ all smooth subregions $\}$

general $\Phi-$

$\Phi$ unbounded in

some finite neigh- : $S O_{H \Phi}=S O_{H B}$

borhood of $t=0$

$\Phi$ bounded in some

neighborhood of $t=0$

$\Phi$ bounded in any finite
neighborhood of $t=0$
$\limsup _{t \rightarrow \infty} \Phi(t) / t<\infty: S O_{H \Phi}=S O_{H P}$
$\limsup _{t \rightarrow \infty} \Phi(t) / t=\infty: S O_{H \Phi}=S O_{H B}$

REMark. Observe that the manifold structure of $R$ is needed only in the proof of the inclusion $S O_{H P} \subset S O_{H \Phi}$ where Theorem 1 is used. 
All other relations obtained in the diagram are therefore valid for harmonic spaces. It remains an open question whether the relation $S O_{H \Phi}=S O_{H P}$ is itself valid for harmonic spaces.

\section{REFERENCES}

1. M. Nakai, On $\Phi$ :-bounded harmonic functions, Ann. Inst. Fourier (Grenoble) 16 (1966), fasc. 1, 145-157. MR 34 \#2863.

2. - Existence of positive harmonic functions, Proc. Amer. Math. Soc. 17 (1966), 365-367. MR 33 \#5880.

3. W. H. Ow, Wiener's compactification and $\Phi$-bounded harmonic functions in the classification of harmonic spaces, Pacific J. Math. 38 (1971), 759-769.

4. M. Parreau, Sur les moyennes des fonctions harmoniques et analytiques et la classification des surfaces de Riemann, Ann. Inst. Fourier (Grenoble) 3 (1951), 103-197. MR 14, 263.

5. L. Sario and M. Nakai, Classification theory of Riemann surfaces, Die Grundlehren der math. Wissenschaften, Band 164, Springer-Verlag, New York and Berlin, 1970. MR 41 \#8660.

Michigan State University, East Lansing, Michigan 48823 
